\title{
Evaluation of brain injury criteria based on reliability analysis
}

\author{
MÁTÉ HAZAY*, IMRE BOJTÁR \\ Department of Structural Mechanics, Budapest University of Technology and Economics, Budapest, Hungary.
}

\begin{abstract}
Purpose: Among the proposed brain injury metrics, Brain Injury Criteria (BrIC) is a promising tool for performing safety assessment of vehicles in the future. In this paper, the available risk curves of BrIC were re-evaluated with the use of reliability analysis and new risk curves were constructed for different injury types based on literature data of tissue-level tolerances. Moreover, the comparison of different injury metrics and their corresponding risk curves were performed. Methods: Tissue-level uncertainties of the effect and resistance were considered by random variables. The variability of the tissue-level predictors was quantified by the finite element reconstruction of 100 frontal crash tests which were performed in Simulated Injury Monitor environment. The applied tests were scaled to given BrIC magnitudes and the injury probabilities were calculated by Monte Carlo simulations. New risk curves were fitted to the observed results using Weibull and Lognormal distribution functions. Results: The available risk curves of diffuse axonal injury (DAI) could be slightly improved, and combined AIS 4+ risk curves were obtained by considering subdural hematoma and contusion as well. The performance of several injury metrics and their risk curves were evaluated based on the observed correlations with the tissue-level predictors. Conclusions: The cumulative strain damage measure and the BrIC provide the highest correlation $\left(R^{2}=0.61\right)$ and the most reliable risk curve for the evaluation of DAI. Although the observed correlation is smaller for other injury types, the BrIC and the associated reliability analysis-based risk curves seem to provide the best available method for estimating the brain injury risk for frontal crash tests.
\end{abstract}

Key words: traumatic brain injuries, finite element simulations, injury risk curves, frontal car crashes

\section{Introduction}

Previous epidemiological studies revealed that traumatic brain injury (TBI) is a critical health problem worldwide, and motor vehicle crashes are one of the main causes of these injuries [2], [7]. To reduce the number and severity of the crash-related injuries, the design procedure of vehicles is governed by safety standards and public car assessment programs. The safety evaluation of cars is based on specified threshold values of injury metrics which must not be exceeded during the well-defined crash tests. These thresholds are determined by injury risk curves which give the injury probability in the function of the applied injury metric. As the effectiveness of the passive restraint system of cars highly depends on the reliability of the applied evaluation procedure, the advanced numerical modelling of the brain for impact conditions [12], [18], [24] and the determination of reliable metrics and associated risk curves [20], [23] have been a major research goal of the biomechanical studies of TBIs in the previous decades. In terms of their significance, the Head Injury Criterion (HIC) [23] stands out from all previously proposed metric, since it has been used in the standardised evaluation procedures worldwide in the previous decades (e.g., Federal Motor Vehicle Safety Standard (FMVSS 208) [14], European Directive (ECE R94) [22], etc.). In this metric, the loading magnitude is described by a $\mathrm{HIC}$ value which is the maximum of the following integral:

* Corresponding author: Máté Hazay, Department of Structural Mechanics, Budapest University of Technology and Economics, 1111 Budapest, Müegyetem rkp. 3. Building K, mezzanine floor 63 (KM63). Phone: +36 1463 1099, e-mail: hazay.mate@emk.bme.hu Received: November 19th, 2020

Accepted for publication: October 10th, 2021 


$$
\mathrm{HIC}=\left[\frac{1}{t_{2}-t_{1}} \int_{t_{1}}^{t_{2}} a(t) \mathrm{d} t\right]^{2,5}\left(t_{2}-t_{1}\right),
$$

where $t$ is the time, $a(t)$ is the measured resultant instantaneous translational acceleration at the centre of gravity of the head and $t_{2}-t_{1}$ is the time interval chosen to maximize the value of HIC. The FMVSS 208 standard applies a maximum $15 \mathrm{~ms}$ long time interval (in Eq. (1) to obtain a $\mathrm{HIC}_{15}$ value) and a $\mathrm{HIC}_{15}=700$ threshold which is thought to be related to the $5 \%$ probability of a serious (AIS $4+)$ head injury $\left(P_{\text {AIS } 4+}\right)$ [6] according to the original risk curve of Prasad and Mertz [16]:

$$
P_{\mathrm{AIS} 4+}\left(\mathrm{HIC}_{15}\right)=\Phi\left(\frac{\mathrm{HIC}_{15}-134}{430}\right),
$$

where $\Phi$ is the cumulative standard normal distribution function. Although the current standards are still based on HIC, serious doubts exist regarding its reliability due to its approximations and negligence [9]. By now, significant scientific proof exists indicating that the tissue-level predictors have a very small correlation with $\mathrm{HIC}_{15}$, and this correlation can be much better when other head motion-based metrics are applied [4], [20], [25]. Not just the HIC metric itself, but the reliability of the corresponding risk curve (Eq. (2)) is also questionable [9]. Due to the large uncertainties which are present during the application of HIC, previous reliability analysis-based research [9] led to a significantly different risk curve:

$$
P_{\mathrm{AIS} 4+}\left(\mathrm{HIC}_{15}\right)=\Phi\left(\frac{\ln \left(\mathrm{HIC}_{15}\right)-6.5445}{1.993}\right) .
$$

As the doubts regarding the reliability of the HIC metric seems to be justified, the modification of the standardised evaluation procedures is expected. As the National Highway Traffic Safety Administration (NHTSA) has already implemented the Brain Injury Criteria (BrIC) [20] into the New Car Assessment Program (NCAP) [15] to provide an extension beside HIC, BrIC seems to be a potential candidate to become a fundamental brain injury metric in the following decades. In this metric, the magnitude of the effect is quantified by a BrIC value which is given by the maximum directional angular velocities $\left(\omega_{x}, \omega_{y}, \omega_{z}\right)$ of the head:

$$
\operatorname{BrIC}=\sqrt{\left(\frac{\omega_{x}}{\omega_{x C}}\right)^{2}+\left(\frac{\omega_{y}}{\omega_{y C}}\right)^{2}+\left(\frac{\omega_{z}}{\omega_{z C}}\right)^{2}},
$$

where $\omega_{x C}=66.25 \mathrm{rad} / \mathrm{s}, \omega_{y C}=56.45 \mathrm{rad} / \mathrm{s}$ and $\omega_{z C}=$ $42.87 \mathrm{rad} / \mathrm{s}$ are critical maximum angular velocities (which correspond to the $50 \%$ probability of an AIS $4+$ injury) [20]. Corresponding risk curves related to the probability of an AIS 4+ diffuse axonal injury (DAI) $\left(P_{\mathrm{DAI}}\right)$ have been constructed following Weibull distribution [20]:

$$
\begin{gathered}
P_{\mathrm{DAI}}(\mathrm{BrIC})=1-e^{-\left(\frac{\mathrm{BrIC}}{1.204}\right)^{2.84}}, \\
P_{\mathrm{DAI}}(\mathrm{BrIC})=1-e^{-\left(\frac{\mathrm{BrIC}-0.523}{0.647}\right)^{1.8}} .
\end{gathered}
$$

For DAI, two tissue-level predictors have been applied in previous research works [4], [18], [20], [25]: the maximum principal strain (MPS) (Eq. (5a)) and the cumulative strain damage measure (CSDM) associated with a strain threshold of 0.25 (Eq. (5b)).

Previous large-scale correlation studies [4], [20] have shown that BrIC gives a relatively good correlation with the tissue-level predictors (MPS and CSDM). However, the proposed risk curve may need to be reevaluated for eliminating the following setbacks:

- During the construction of Eqs. (5a), (5b), a deterministic linear connection has been assumed between the tissue-level predictors and BrIC [20]. However, different tissue-level outcomes can occur in different crashes even if they represent the same BrIC value. Such tissue-level uncertainty of the effect (and the resistance also) can influence the injury probability which should be considered during the construction of risk curves.

- The available risk curves are based on the consideration of DAI only [20], while focal injury types, such as acute subdural hematoma (ASDH) and contusion, were not taken into account.

- Although the CSDM seemed to be a better predictor than MPS in a previous study [20], its corresponding risk curve (Eq. (5b)) has the disadvantage that it gives zero injury probability in the BrIC $=0-0.523$ interval, which partially comes from the negligence of the above-mentioned tissue-level uncertainties. A more reliable estimation may be needed in the $\mathrm{BrIC} \approx 0.3-0.55$ "design interval" because it is possible that a new threshold value related to a combined AIS 4+ head injury belongs to this interval (assuming that it will be calibrated to the $5 \%$ injury probability as earlier).

In this study, the re-evaluation of the available risk curves (Eqs. (5a), (5b)) is performed by tissue-level reliability analysis using finite element (FE) reconstruction of frontal crash tests. In this analysis, some of the most important uncertainties are taken into account by random variables, while each above-noted injury type is considered by a separate limit-state function [9]. 
Once the reliability-based reconstruction of the BrIC-related risk curves is accomplished, the comparison of several injury metrics and risk curves is performed. The selection of the analysed injury metrics is governed by the following aspects:

- besides translational acceleration and rotational velocity-based metrics (HIC and BrIC), angular acceleration-based metrics should be analysed also;

- only those metrics are considered which gave a high degree of correlation with the tissue-level predictors in previous comparison studies [4], [25];

- an injury risk curve is available for the given metric (or at least its estimation is possible based on the available data).

Based on these considerations, another analysed metric is the Rotational Injury Criterion (RIC) [11]. It is formulated similarly as the HIC, but here the translational acceleration (Eq. (1)) is replaced with the rotational acceleration $(\alpha)$ :

$$
\mathrm{RIC}=\left[\frac{1}{t_{2}-t_{1}} \int_{t_{1}}^{t_{2}} \alpha(t) \mathrm{d} t\right]^{2.5}\left(t_{2}-t_{1}\right)
$$

and a $t_{2}-t_{1} \leq 36 \mathrm{~ms}$ time interval was proposed [11]. For RIC, only a risk curve related to the probability of an AIS $2+$ injury $\left(P_{\mathrm{AIS} 2+}\right)$ is available [1], [11]:

$$
P_{\mathrm{AIS} 2+}(\mathrm{RIC})=\frac{1}{1+e^{7.036-0.000000679 \mathrm{RIC}}} .
$$

Furthermore, the Rotational Velocity Change Index ( $\mathrm{RVCI}$ ) is also considered, which is given by the following equation [25]:

$$
\mathrm{RVCI}=\sqrt{R_{x}\left(\int_{t_{1}}^{t_{2}} \alpha_{x} \mathrm{~d} t\right)^{2}+R_{y}\left(\int_{t_{1}}^{t_{2}} \alpha_{y} \mathrm{~d} t\right)^{2}+R_{z}\left(\int_{t_{1}}^{t_{2}} \alpha_{z} \mathrm{~d} t\right)^{2}},
$$

where $R_{x}, R_{y}$ and $R_{z}$ are weighting factors (detailed in [25] for CSDM and MPS separately) and $\alpha_{x}, \alpha_{y}, \alpha_{z}$ are the components of the instantaneous rotational acceleration about each orthogonal direction and $t_{2}-t_{1} \leq 10 \mathrm{~ms}$ is the time interval which is chosen to maximize the value of RVCI [25]. No injury risk curve has been proposed related to RVCI [1], however, the following RVCI-MPS relation was observed (which is used to obtain an approximate risk curve as detailed in the Methods section):

$$
\text { MPS }=0.0162 \mathrm{RVCI}+0.0855 \text {. }
$$

Following the above-mentioned goals, three hypotheses are analysed in this study:

(1) With the consideration of DAI only, the obtained new risk curves via reliability analysis gives larger injury probabilities in the "design interval" than Eqs. $(5 \mathrm{a}, 5 \mathrm{~b})$, however, these probabilities have a similar magnitude to those which can be obtained by the available risk curves.

(2) With the consideration of ASDH and contusion also, significantly larger probabilities are obtained for a combined AIS 4+ injury.

(3) Related to DAI, the new CSDM-based risk curve (determined by reliability analysis) gives the closest results to the probabilities calculated by tissuelevel metrics.

\section{Materials and methods}

In this study, the tissue-level predictors are determined by $\mathrm{FE}$ reconstruction of standardised frontal crash tests [14] where Hybrid III adult male dummies were applied (Fig. 1a). The dummy's head is instrumented with the Nine Accelerometer Array Package (NAAP) (Fig. 1a) [19] and the measured acceleration records are downloaded from the NHTSA database. Based on the rigid body motion of the dummy's head [19], loading curves (including the translational and rotational velocities of the head) (Fig. 1b) are calculated from the measured acceleration-time histories using the Simulated Injury Monitor (SIMon) software [17], [18]. Following previous impact biomechanical studies of blunt trauma [4], [9], [20], FE simulations are performed on previously validated head models of the SIMon software [17], [18]. The improved SIMon model is considered as one of the currently available state-of-art models, which was also applied to construct BrIC metric and its corresponding risk curves (Eqs. (5a), (5b)) [20]. With the obtained loading curves, the motion of the dummy's head is applied to the model's rigid skull as a prescribed boundary motion [17]. Due to the movement of the skull, the impact energy is transferred to the brain via contact algorithms between the skull and the brain (detailed in [17], [18]). Following the recommendations of the authors [18], the DAI-related tissue-level predictors (MPS and CSDM) are determined by the improved SIMon model (Fig. 1c), while the relative motion damage measure (RMDM) for characterizing ASDH and the dilatation damage measure (DDM) for the analysis of contusion are calculated by the standard model (Fig. 1c) [17]. 


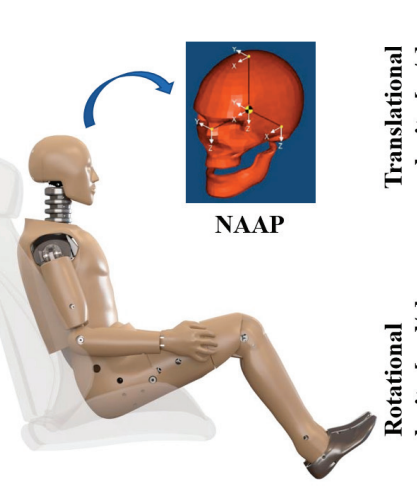

a) Hybrid III dummy
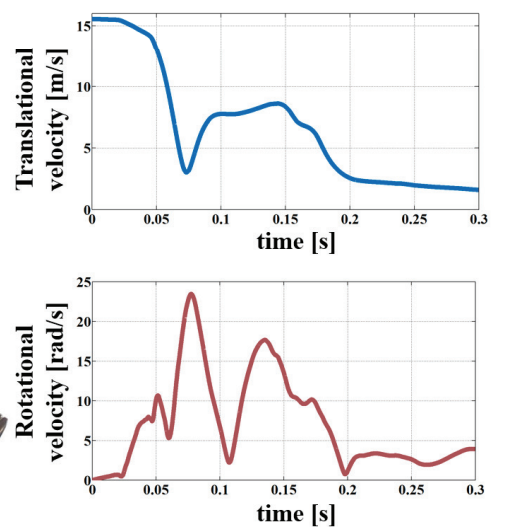

b) Resultant head velocities
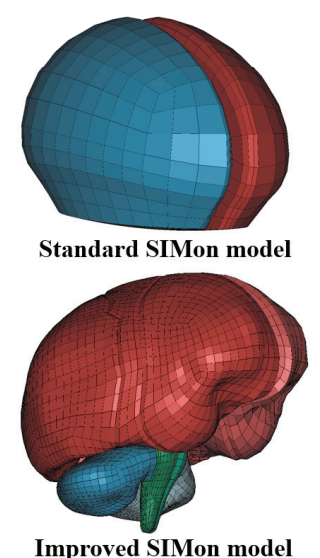

c) FE models

Fig. 1. Framework of the FE reconstruction of frontal crash tests: a) crash tests with dummies instrumented with NAAP; b) resultant translational and rotational velocities of the head; c) FE head models

The basic goal of the reliability analysis is to determine the injury probability for given BrIC values with the consideration of some of the most important types of tissue-level uncertainties. In this analysis, each considered injury type is represented by a $G_{i}(i=1, \ldots, 4)$ limitstate function (where $i=1$ and $i=2$ belong to DAI with the MPS and CSDM-based description, respectively), $i=3$ belongs to ASDH and $i=4$ belongs to contusion):

$$
\begin{gathered}
G_{1}=R_{\mathrm{MPS}}-E_{\mathrm{MPS}}, \\
G_{2}=R_{\mathrm{CSDM}}-E_{\mathrm{CSDM}},
\end{gathered}
$$

$$
\begin{gathered}
G_{3}=R_{\mathrm{RMDM}}-E_{\mathrm{RMDM}}, \\
G_{4}=R_{\mathrm{DDM}}-E_{\mathrm{DDM}} .
\end{gathered}
$$

The injury risk is quantified as the probability that the considered limit-state function gives a negative value for a random realization of the corresponding random variables. Limit-state functions include random variables which are intended to describe uncertainties of the tissue-level effect ( $E_{\mathrm{MPS}}, E_{\mathrm{CSDM}}$, $\left.E_{\mathrm{RMDM}}, E_{\mathrm{DDM}}\right)$ and the resistance $\left(R_{\mathrm{MPS}}, R_{\mathrm{CSDM}}, R_{\mathrm{RMDM}}\right.$, $R_{\mathrm{DDM}}$ ).

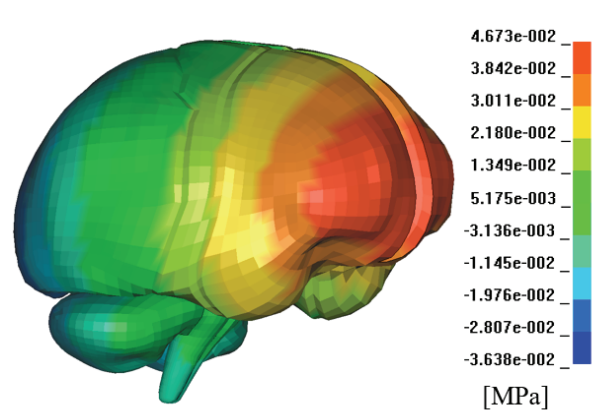

a) Pressure at $\mathrm{BrIC}=0.5$

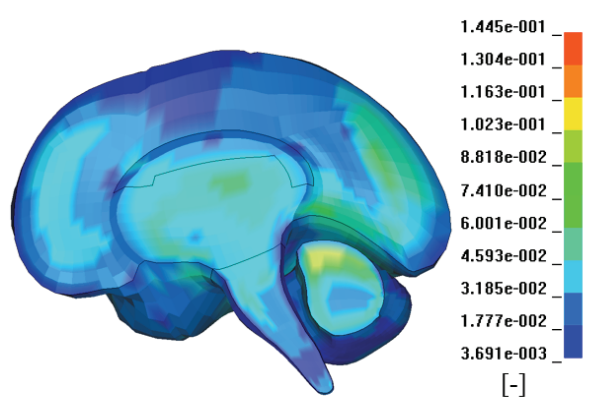

c) $1^{\text {st }}$ principal strain at $B r I C=0.5$

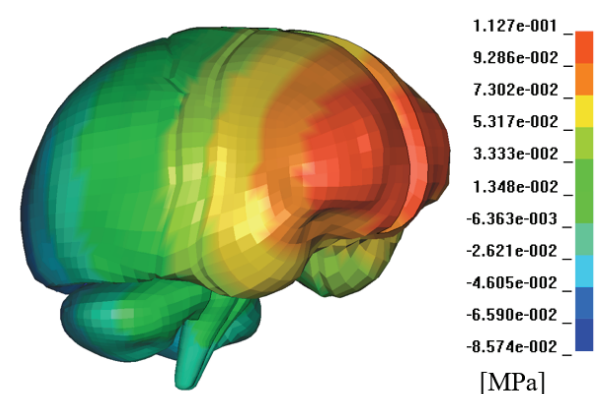

b) Pressure at $\mathrm{BrIC}=1.25$

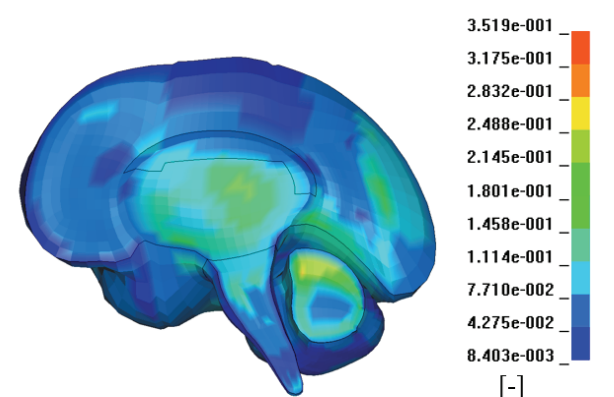

d) $1^{\text {st }}$ principal strain at $B r I C=1.25$

Fig. 2. Illustration of FE results for different loading intensity: a) pressure at $\mathrm{BrIC}=0.5$, b) pressure at $\mathrm{BrIC}=1.25, \mathrm{c}) 1$ st principal strains at $\mathrm{BrIC}=0.5, \mathrm{~d}) 1 \mathrm{st}$ principal strains at $\mathrm{BrIC}=1.25$ 
In general, a rigid body motion-based metric always contains a considerable uncertainty because such a parameter cannot perfectly predict the complicated highly nonlinear behaviour of the brain tissue. This can be observed if multiple crashes are reconstructed which belong to the same value of the applied metric, the obtained tissue-level predictors will show a certain variability. This record-to-record variability should be considered during the construction of the risk curves as previous reliability-based evaluation of HIC proved that it can have a significant contribution to the injury probability [9]. To quantify this variability, $100 \mathrm{FE}$ simulations are performed for given $\mathrm{BrIC}$ values $(0.2,0.5,0.7$, $0.85,1.0,1.25$ and 1.5) (i.e., in total, 700 simulations are completed with the improved and 700 simulations with the standard SIMon model). The test selection from the NHTSA database was governed by the following criteria:

- frontal crash tests with a rigid barrier (as per FMVSS 208) are analysed;

- the impact velocity is $56 \mathrm{~km} / \mathrm{h}$ (standard value as per FMVSS 208 and ECE R94);

- airbag and safety belt are applied;

- a Hybrid III adult male dummy (instrumented with NAAP) is used at the driver's seat.

To obtain multiple realistic crash records which represent the same BrIC value, the nine accelero-

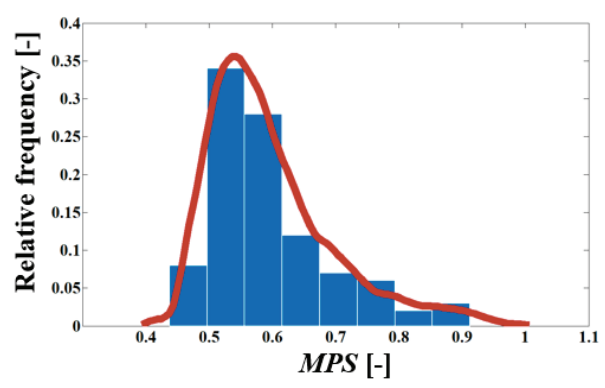

a) Fitting distribution function to $\mathrm{FE}$ results

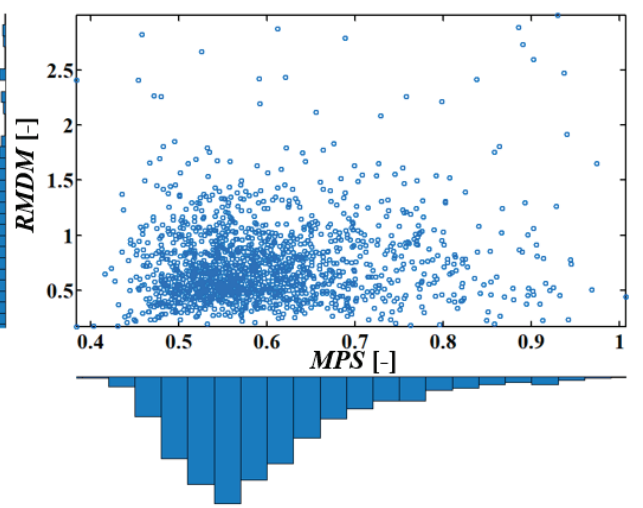

c) Generating correlated samples of the tissue-level effects

Fig. 3. Main steps of reliability analysis: a) fitting distribution function to FE results, b) Monte Carlo simulation, c) Generating correlated samples with given marginal distributions

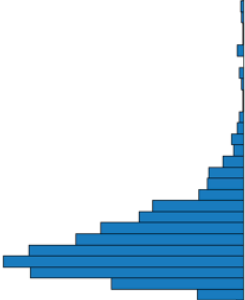

grams of these tests are scaled linearly by a mutual scaling factor.

Once the 100 realizations of the tissue-level predictors are obtained from the simulations (briefly illustrated in Fig. 2), random variables describing the effect in Eqs. (10a)-(10d) are fitted to the observed data by the log-likelihood method in Matlab [21] using non-parametric Kernel distribution function with Epanechnikov kernels [3] (Fig. 3a).

The available data related to the tissue-level resistance also show a considerable variability. Following the results of previous regression analysis of scaled animal data [17], [20], the random variables of tissue-level resistance can be characterized as it is summarized in Table 1.

Table 1. Random variables of tissue-level resistance

\begin{tabular}{|c|c|c|}
\hline $\begin{array}{c}\text { Random } \\
\text { variable }\end{array}$ & Distribution function & Reference \\
\hline$R_{\mathrm{MPS}}$ & $F(x)=P\left(R_{\mathrm{MPS}}<x\right)=1-e^{-\left(\frac{x}{1.01}\right)^{2.84}}$ & {$[20]$} \\
\hline$R_{\mathrm{CSDM}}$ & $F(x)=P\left(R_{\mathrm{CSDM}}<x\right)=1-e^{-\left(\frac{x}{0.60}\right)^{1.80}}$ & {$[20]$} \\
\hline$R_{\mathrm{RMDM}}$ & $F(x)=P\left(R_{\mathrm{RMDM}}<x\right)=\Phi\left(\frac{x-1}{0.35}\right)$ & {$[17]$} \\
\hline$R_{\mathrm{DDM}}$ & $F(x)=P\left(R_{\mathrm{DDM}}<x\right)=\Phi\left(\frac{\ln (x)+2.8811}{0.7072}\right)$ & {$[17]$} \\
\hline
\end{tabular}

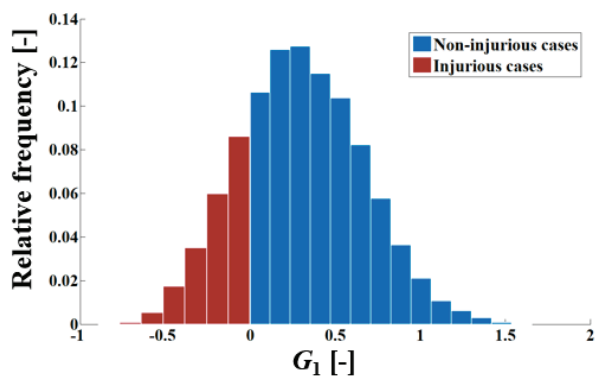

b) Monte Carlo simulation: outcome of experiments 
The injury probability is calculated for each injury type with Monte Carlo simulations by generating $10^{6}$ random samples of the random variables. The limitstate functions are evaluated for all sample pairs, and the number of the injurious cases (i.e., where the limitstate function gives a negative value) is counted. Finally, the total number of the injurious cases is divided by the total number of samples to obtain the injury probability (Fig. 3b).

After determining the injury probability for each injury type, the combined AIS 4+ injury probability shall be determined. As the occurrence of any of the above-mentioned injury types already causes an AIS 4+ injury, these injury types form a so-called series system. The reliability of the whole system is also evaluated by Monte Carlo simulations, where the generated random vectors of the effect and the resistance have three components (i.e., one for each injury type). To take the correlation of the different injury types into account, the random vector of the effect is generated by considering the observed correlation of MPS (or CSDM), RMDM, and DDM results. This correlation is described by the Kendall-type rank correlation coefficient, and the correlated vector components of the effect are generated by multivariate copula functions whose marginal distributions equal to the Kernel-type distributions of the corresponding random variables (Fig. 3c).

Once the injury probabilities are obtained for the analysed BrIC values, new risk curves are constructed by the least-square method using Weibull and Lognormal distribution functions (whichever gives the better approximation in terms of the sum of squared errors (SSE)).

Once the reliability-based evaluation of the BrIC-related risk curves is completed, the comparison of several injury criteria is performed by the numerical reconstruction of the previously selected 100 frontal crash tests with their original, unscaled loading curves. This comparison includes two separate investigations:

1. Following a traditional approach [4], [20], [25], the adequacy of the selected injury metrics is evaluated by their degree of correlation with the tissue-level predictors.

2. Besides the formulation of the available metrics, the reliability of their corresponding risk curves is also analysed. Since a good injury metric should reliably bridge the gap between the rigid body motion of the head and the tissue-level predictors, it is analysed which injury risk curve gives the best approximation (in terms of SSE) of the results obtained by tissue-level predictors.
In order to get a reasonable approximation of the AIS 4+ curve related to RIC for the present comparison study, the available AIS 2+ risk curve (Eq. (7)) is scaled by the method which previously led to the BrIC-related risk curves of different injury severities [20] (following the recommendations of NHTSA [13]). In this approach, the median point of the Weibull distribution function is scaled by the $\beta_{24}=2.0$ factor [20] while keeping the shape parameter constant. To follow this approach as close as possible, an alternative AIS 2+ risk curve with Weibull distribution (with $\alpha=1.1367 \cdot 10^{7}$ scale parameter and $k=4.1965$ shape parameter) is fitted to the available risk curve (Eq. (7)) exploiting that only minor differences can be observed between these curves. Afterwards, this curve is scaled by $\beta_{24}$ to obtain the following AIS $4+$ curve:

$$
P_{\mathrm{DAI}}(\mathrm{RIC})=1-e^{-\left(\frac{\mathrm{RIC}}{2.27334 \cdot 10^{7}}\right)^{4.196}} .
$$

For the RVCI, an AIS 4+ risk curve is not available either, however, in the knowledge of the MPS-RVCI relation described in Eq. (9), the original MPS-related AIS 4+ tissue-level tolerance curve (Table 1) can be expressed in the function of RVCI to obtain the following estimation:

$$
P_{\mathrm{DAI}}(\mathrm{RVCI})=1-e^{-\left(\frac{0.0086 \cdot \mathrm{RVCl}-0.1439}{0.6}\right)^{1.8}} .
$$

\section{Results}

Statistical parameters of the 100 realizations of the tissue-level effects obtained by FE simulations are summarized in Table 2.

Injury probabilities at given BrIC values obtained by Monte Carlo simulations are shown in Table 3 (where $P_{\text {DAI(MPS) }}$ is the probability of DAI based on Eq. (10a), $P_{\text {DAI(CSDM) }}$ is the probability of DAI based on Eq. (10b), $P_{\mathrm{ASDH}}$ is the probability of ASDH, $P_{\text {Contusion }}$ is the probability of contusion, while $P_{\mathrm{AIS} 4+(\mathrm{MPS})}$ and $P_{\mathrm{AIS}}$ ${ }_{4+(\mathrm{CSDM})}$ are the combined injury probabilities following an MPS-based and a CSDM-based description of DAI, respectively).

Based on the result of Table 3, new risk curves are constructed by curve fitting (given in Eqs. (13a), (13b) related to DAI and in Eqs. (14a), (14b) related to ASDH and contusion, respectively).

$$
P_{\mathrm{DAI}(\mathrm{MPS})}(\mathrm{BrIC})=1-e^{-\left(\frac{\mathrm{BrIC}}{1.4283}\right)^{2.0348}},
$$




$$
\begin{gathered}
P_{\mathrm{DAI}(\mathrm{CSDM})}(\mathrm{BrIC})=\Phi\left(\frac{\ln (\mathrm{BrIC})-0.0957}{0.3556}\right), \\
P_{\mathrm{ASDH}}(\mathrm{BrIC})=1-e^{-\left(\frac{\mathrm{BrIC}}{1.1078}\right)^{2.5778}}, \\
P_{\text {Contusion }}(\mathrm{BrIC})=1-e^{-\left(\frac{\mathrm{BrIC}}{2.0340}\right)^{4.8738}} .
\end{gathered}
$$

Following the above-noted curve fitting procedure, new risk curves are obtained related to a combined AIS4+ injury. In Equation (15a), the $P_{\text {AIS } 4+}$ risk curve is given by considering the MPS-based description of
DAI (Eq. (10a)), while in the construction of Eq. (15b), the CSDM-related limit-state function (Eq. (10b)) is applied.

$$
\begin{gathered}
P_{\mathrm{AIS} 4+(\mathrm{MPS})}(\mathrm{BrIC})=1-e^{-\left(\frac{\mathrm{BrIC}}{0.9048}\right)^{2.2944}}, \\
P_{\mathrm{AIS} 4+(\mathrm{CSDM})}(\mathrm{BrIC})=1-e^{-\left(\frac{\mathrm{BrIC}}{0.9435}\right)^{3.1570}} .
\end{gathered}
$$

Risk curves related to DAI alongside with the avail-

\begin{tabular}{|c|c|c|c|c|c|c|c|}
\hline Effect & BrIC & $\mu$ & $\sigma$ & $\mathrm{CoV}$ & $\eta$ & $\kappa$ & $r$ \\
\hline MPS & \multirow{4}{*}{0.20} & 0.193 & 0.034 & 0.174 & 1.331 & 5.129 & 0.180 \\
\hline CSDM & & $\approx 0$ & - & - & - & - & - \\
\hline RMDM & & 0.186 & 0.085 & 0.460 & 2.173 & 10.554 & 0.583 \\
\hline DDM & & $\approx 0$ & - & - & - & - & - \\
\hline MPS & \multirow{4}{*}{0.50} & 0.450 & 0.076 & 0.170 & 1.212 & 4.300 & 0.397 \\
\hline CSDM & & 0.033 & 0.046 & 1.385 & 3.737 & 21.372 & 0.337 \\
\hline RMDM & & 0.516 & 0.224 & 0.435 & 1.795 & 7.978 & 1.428 \\
\hline DDM & & 0.00030 & 0.00108 & 3.564 & 5.096 & 29.499 & 0.00748 \\
\hline MPS & \multirow{4}{*}{0.70} & 0.596 & 0.096 & 0.161 & 1.246 & 4.323 & 0.475 \\
\hline CSDM & & 0.145 & 0.105 & 0.728 & 2.411 & 9.982 & 0.627 \\
\hline RMDM & & 0.727 & 0.301 & 0.413 & 2.038 & 10.709 & 2.105 \\
\hline DDM & & 0.00147 & 0.00392 & 2.666 & 3.359 & 13.457 & 0.02079 \\
\hline MPS & \multirow{4}{*}{0.85} & 0.691 & 0.106 & 0.154 & 1.157 & 4.028 & 0.481 \\
\hline CSDM & & 0.268 & 0.131 & 0.488 & 1.753 & 6.560 & 0.706 \\
\hline RMDM & & 0.909 & 0.390 & 0.429 & 2.062 & 10.199 & 2.661 \\
\hline DDM & & 0.00374 & 0.00794 & 2.123 & 3.255 & 13.634 & 0.04589 \\
\hline MPS & \multirow{4}{*}{1.00} & 0.795 & 0.153 & 0.192 & 3.165 & 19.676 & 1.192 \\
\hline CSDM & & 0.419 & 0.150 & 0.359 & 1.194 & 5.057 & 0.803 \\
\hline RMDM & & 1.128 & 0.485 & 0.430 & 1.964 & 8.829 & 2.989 \\
\hline DDM & & 0.00770 & 0.01342 & 1.744 & 3.852 & 21.227 & 0.09499 \\
\hline MPS & \multirow{4}{*}{1.25} & 0.919 & 0.132 & 0.144 & 1.067 & 5.953 & 0.924 \\
\hline CSDM & & 0.629 & 0.121 & 0.192 & 0.038 & 3.124 & 0.631 \\
\hline RMDM & & 1.423 & 0.551 & 0.387 & 1.225 & 5.522 & 3.431 \\
\hline DDM & & 0.01542 & 0.01609 & 1.044 & 2.149 & 7.755 & 0.07843 \\
\hline MPS & \multirow{4}{*}{1.50} & 1.057 & 0.148 & 0.140 & 1.165 & 5.157 & 0.899 \\
\hline CSDM & & 0.781 & 0.081 & 0.104 & -0.312 & 2.863 & 0.355 \\
\hline RMDM & & 1.818 & 0.593 & 0.326 & 1.164 & 4.816 & 3.383 \\
\hline DDM & & 0.02968 & 0.02016 & 0.679 & 1.561 & 6.006 & 0.10844 \\
\hline
\end{tabular}
able risk curves of Takhounts et al. [20] (Eqs. (5a), (5b)) are shown in Fig. 4.

\begin{tabular}{|c|c|c|c|c|c|c|}
\hline BrIC & $P_{\text {DAI(MPS) }}$ & $P_{\mathrm{DAI}(\mathrm{CSDM})}$ & $P_{\mathrm{ASDH}}$ & $P_{\text {Contusion }}$ & $P_{\mathrm{AIS4+(MPS)}}$ & $P_{\mathrm{AIS} 4+(\mathrm{CSDM})}$ \\
\hline $\begin{array}{c}0.20 \\
0\end{array}$ & 0.010 & 0.000 & 0.013 & 0.000 & 0.025 & 0.013 \\
\hline 0.50 & 0.102 & 0.013 & 0.121 & 0.000 & 0.224 & 0.130 \\
\hline 0.70 & 0.208 & 0.094 & 0.259 & 0.004 & 0.425 & 0.313 \\
\hline 0.85 & 0.296 & 0.223 & 0.396 & 0.017 & 0.581 & 0.511 \\
\hline 1.00 & 0.395 & 0.409 & 0.549 & 0.036 & 0.724 & 0.716 \\
\hline 1.25 & 0.530 & 0.649 & 0.729 & 0.082 & 0.864 & 0.899 \\
\hline 1.50 & 0.665 & 0.792 & 0.896 & 0.202 & 0.963 & 0.982 \\
\hline
\end{tabular}

Table 2. Statistical parameters of $F E$ results $(\mu-$ mean value, $\sigma$-standard deviation, $\mathrm{CoV}$ - coefficient of variation, $\eta$ - skewness, $\kappa$ - kurtosis, $r-$ range)

Table 3. Injury probabilities obtained by reliability analysis 


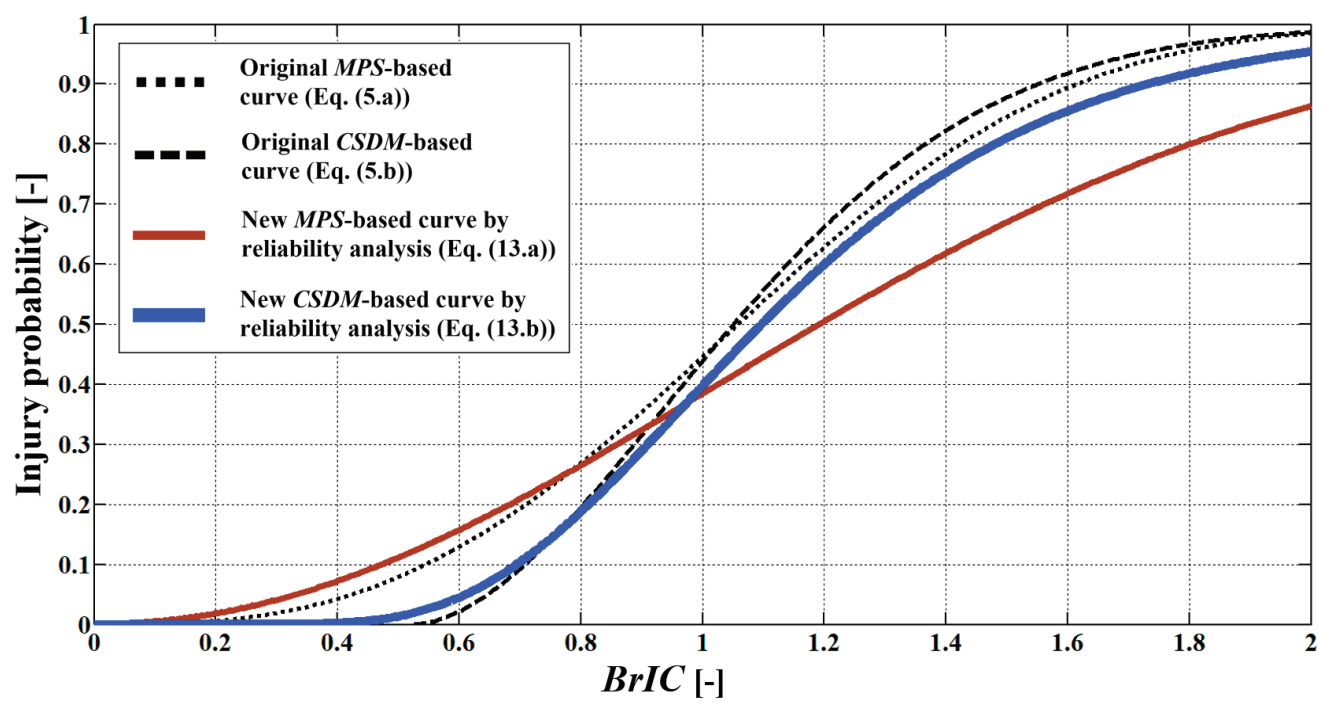

Fig. 4. Risk curves related to DAI

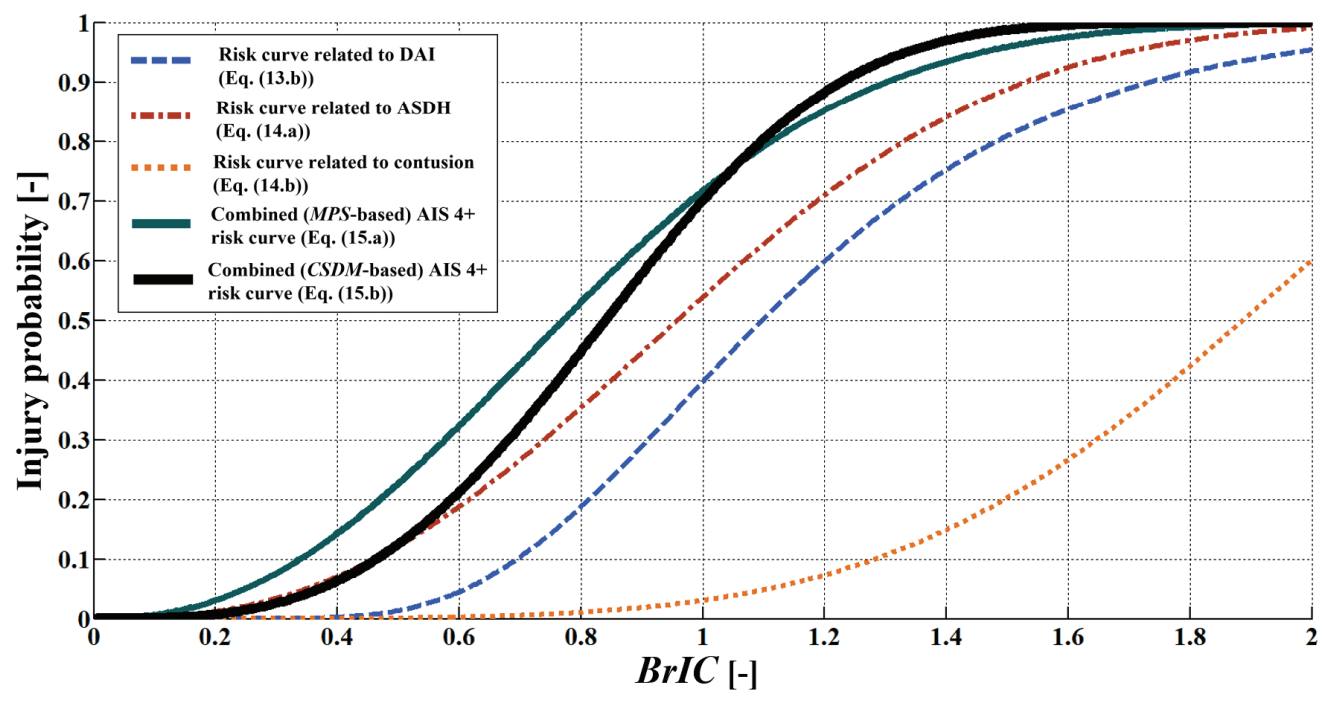

Fig. 5. Risk curves related to different injury types

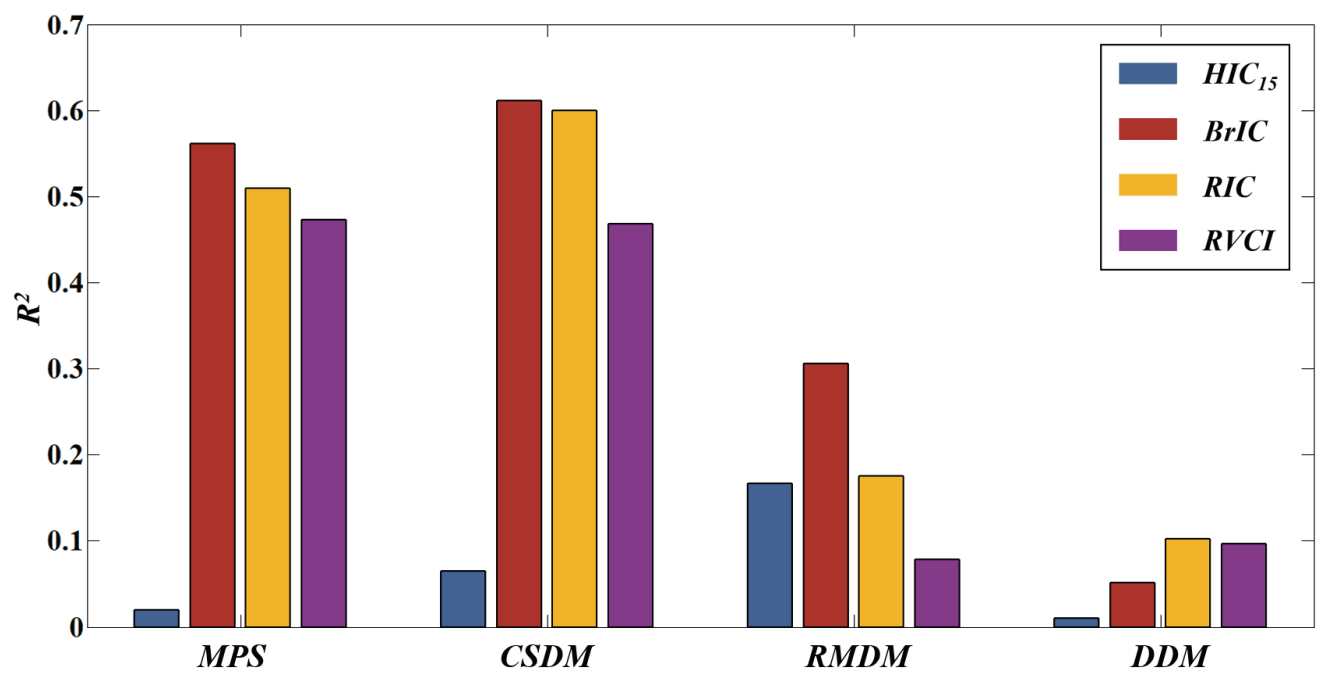

Fig. 6. Correlation of injury metrics with tissue-level predictors 
Risk curves related to different injury types and the combined AIS 4+ brain injury are shown in Fig. 5.

The correlation (in terms of the coefficient of determination $\left(R^{2}\right)$ ) between the analysed injury metrics and the tissue-level predictors are shown in Fig. 6.
The obtained discrepancy (expressed by SSE) between the results of the DAI-related injury risk curves and the tissue-level predictors (related to 100 tests) are summarized in Table 4.

The performance of risk curves related to different injury types is summarized in Table 5 .

Table 4. Performance of risk curves related to DAI (bold values were obtained by reliability analysis)

\begin{tabular}{|c|c|c|c|c|c|c|}
\hline & $\begin{array}{c}\text { Takhounts } \\
\text { (MPS) } \\
\text { (Eq. 5a) }\end{array}$ & $\begin{array}{c}\text { Reliability } \\
\text { analysis } \\
\text { (MPS) } \\
\text { (Eq. 13a) }\end{array}$ & $\begin{array}{c}\text { Takhounts } \\
\text { (CSDM) } \\
\text { (Eq. 5b) }\end{array}$ & $\begin{array}{c}\text { Reliability } \\
\text { analysis } \\
\text { (CSDM) } \\
\text { (Eq. 13b) }\end{array}$ & $\begin{array}{c}\text { RIC-based } \\
\text { (Eq. 11) }\end{array}$ & $\begin{array}{c}\text { RVCI-based } \\
\text { (Eq. 12) }\end{array}$ \\
\hline SSE & 0.5093 & $\mathbf{0 . 4 6 1 5}$ & 0.3493 & $\mathbf{0 . 3 1 9 6}$ & 3.3116 & 1.0249 \\
\hline
\end{tabular}

Table 5. Performance of risk curves related to different injury types (bold values were obtained by reliability analysis)

\begin{tabular}{|c|c|c|c|c|c|c|}
\hline & $\begin{array}{c}\text { DAI } \\
(\text { Eq. 13b) }\end{array}$ & $\begin{array}{c}\text { ASDH } \\
(\text { Eq. 14a) }\end{array}$ & $\begin{array}{c}\text { Contusion } \\
\text { (Eq. 14 b) }\end{array}$ & $\begin{array}{c}\text { AIS 4+ } \\
\text { HIC-based } \\
\text { (Eq. 2) }\end{array}$ & $\begin{array}{c}\text { AIS 4+ } \\
\text { HIC-based } \\
\text { (Eq. 3) }\end{array}$ & $\begin{array}{c}\text { AIS 4+ } \\
\text { BrIC-based } \\
\text { (Eq. 15b) }\end{array}$ \\
\hline SSE & $\mathbf{0 . 3 1 9 6}$ & $\mathbf{4 . 2 3 7 9}$ & $\mathbf{0 . 0 0 7 4}$ & 11.672 & $\mathbf{5 . 6 1 9 1}$ & $\mathbf{4 . 0 7 6 9}$ \\
\hline
\end{tabular}

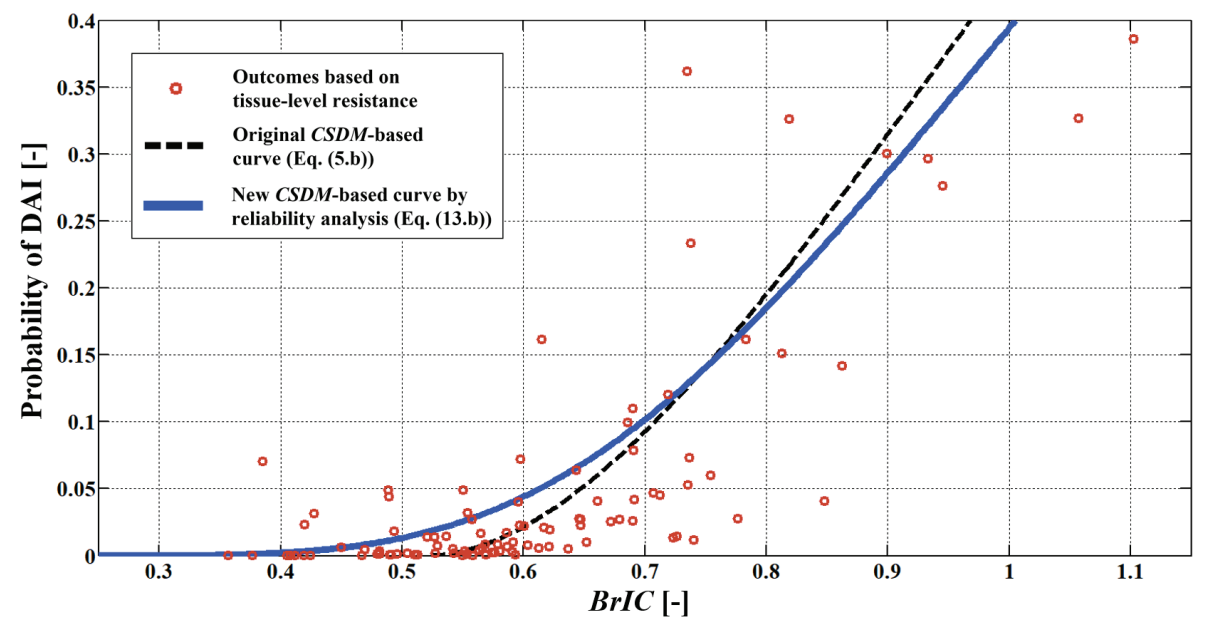

Fig. 7. DAI-related (CSDM-based) risk curves and the outcomes of the 100 reconstructions

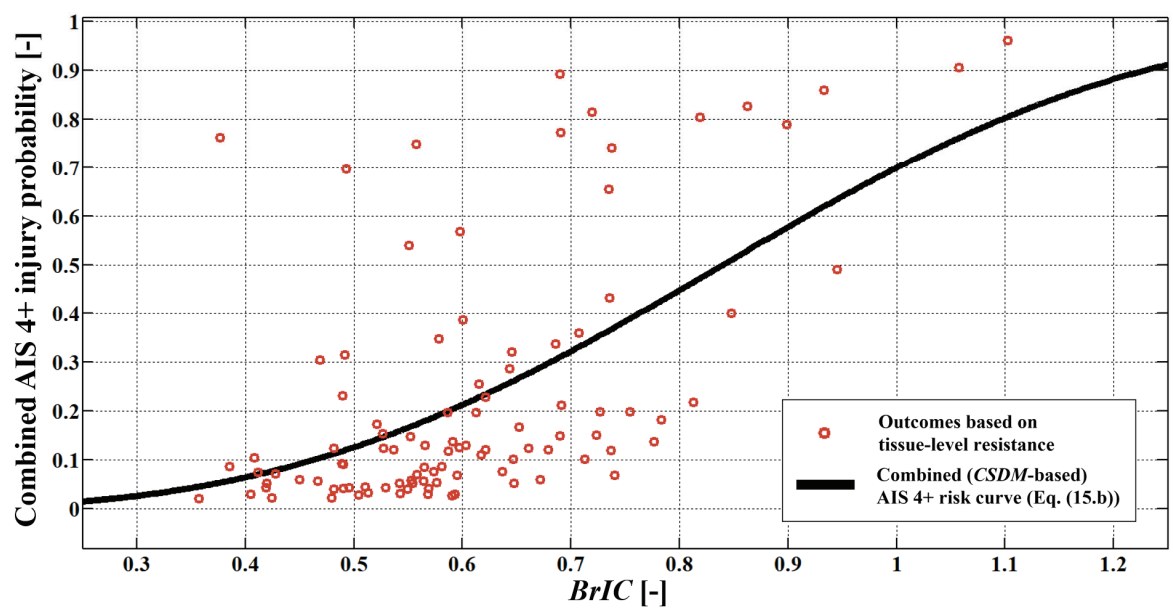

Fig. 8. Combined AIS 4+ injury risk curve and the outcomes of the 100 reconstructions 
The CSDM-based risk curves of DAI and the combined AIS 4+ injury are shown with the outcomes (calculated by tissue-level predictors) of the 100 tests in Figs. 7 and 8.

\section{Discussion}

In this paper, the reliability analysis-based evaluation of the BrIC head injury metric was performed using FE reconstruction of frontal crash tests. The currently available DAI-related risk curves were re-evaluated and slightly modified, and new risk curves were proposed related to ASDH, contusion and the combined AIS 4+ injury. Furthermore, some of the most promising injury metrics and their related injury risk curves were compared to determine which available metric and risk curve seem to be the most reliable tool for estimating the brain injury risk in case of frontal car crashes.

The reliability analysis of DAI resulted in new risk curves (Eqs. (13a), (13b)) which are in relatively good agreement with the available risk curves of Takhounts et al. (Eqs. (5a), (5b)) [20]. Although relatively small discrepancies were observed (Fig. 4), the new risk curves give larger probabilities in the "design interval" which leads to the conclusion that the first hypothesis is true. The main reason behind these discrepancies is that some of the most important uncertainties of the tissue-level effects and resistances were taken into account during the reliability analysis. In a previous reliability-based study of brain injuries [9], it could be observed that the influence of the tissue-level uncertainties on the injury probabilities is primarily governed by the variability of the obtained tissue-level predictors (which can be characterized by $\mathrm{CoV}$ ). From the obtained FE result (Table 2), a $\mathrm{CoV}=0.14-0.192$ could be observed for MPS and a $\mathrm{CoV}=0.104-1.385$ for $\mathrm{CSDM}(\mathrm{CoV}=0.104-0.728$ in the $\mathrm{BrIC}>0.523$ interval where the original risk curve (Eq. (5b)) gives non-zero values). Although these uncertainties are considerable, they are not large enough to cause so significant discrepancy with respect to the original risk curves (Eqs. (5a), (5b)) which was the case in the previous reliability study of the HIC metric [9]). Despite the minor differences, we suggest using the new curves (Eqs. (13a), (13b)) for DAI, because in the reliability analysis the stochastic nature of the tissuelevel predictors was considered. Furthermore, a very convenient result of the reliability analysis is that it could characterize the small injury probabilities even in the BrIC $<0.523$ interval, thus the new, CSDM- related risk curve (Eq. (13b)) gives non-zero values in the $\mathrm{BrIC} \approx 0.3-0.55$ "design interval" which could be a key aspect during the determination of a new threshold value in the future.

The reliability analysis of ASDH and contusion resulted in new risk curves (Fig. 5) which are given by Eqs. (14a), (14b). Based on the comparison of different injury types, a tendency could be observed that the contusion has a significantly smaller probability than the other two types of injuries. This relation seems to be in contradiction with previous real-world observation [10] where the number of contusions reached a similar magnitude than the other two injury types. This discrepancy highlights that the results of such numerical studies are based on the applied tissue-level thresholds which were determined in previous studies [17], [20] using the results of animal experiments and their scaling [17]. Since the current results depend on the applied thresholds, the obtained risk curves should be continuously revised when new experimental studies or real-world observations become available. In this regard, the applied framework of the reliability analysis may not need to be changed during the possible future revision of these results.

The reliability analysis of all injury types resulted in the combined AIS 4+ risk curves (Eqs. (15a), (15b) and Fig. 5) that show considerably larger injury risks than the original DAI-related curves (Eqs. (5a), (5b)). This leads to the conclusion that the second hypothesis is also true.

In the second part of our paper, a comparison study was performed which mainly focuses on the comparison of the available risk curves rather than the comparison of the injury metrics (which has been already investigated in previous studies [4], [25] for DAI). Nevertheless, the obtained correlations (in terms of $R^{2}$ ) of the injury metrics with the tissue-level predictors are shown in Fig. 6 as it provides new results related to the correlation of BrIC with RMDM and DDM, and also gives the possibility to compare our results related to DAI with the observations of previous studies [4], [25]. Our results show that with the DAI-related metrics BrIC gives the best correlation $\left(R^{2}=0.56\right.$ for MPS and $R^{2}=0.61$ for CSDM), while RIC gives the second $\left(R^{2}=0.51\right.$ for MPS and $R^{2}=0.60$ for CSDM) and RVCI gives the third $\left(R^{2}=0.47\right.$ for MPS and $R^{2}=0.47$ for CSDM) largest degree of correlation. This indicates that the BrIC still seems to be the most reliable metric for characterizing DAI in case of frontal crashes. These results coincide with the observation of Gabler et al. [4], although they observed a little stronger correlation $\left(R^{2} \approx 0.7\right)$ with the application of the GHBMC model [5]. However, in another previous 
study [25] RVCI gave a higher degree of correlation which may be attributed to the differences in the applied FE model and that different (small overlap and oblique) crash types were also considered there. In Fig. 6, it could be also observed that HIC gives negligible correlation with tissue-level predictors $\left(R^{2}=0.02\right.$ for MPS and $R^{2}=0.06$ for CSDM) which also agrees with previous observations of Gabler et al. [4].

The correlation analysis with the RMDM metric (Fig. 6) shows that the BrIC still gives the highest degree of correlation $\left(R^{2}=0.31\right)$, but it is much smaller than those which were observed for the DAI-related metrics, indicating that the application of $\mathrm{BrIC}$ is not as reliable for the analysis of ASDH as for the evaluation of DAI. The correlation of each analysed injury metric with DDM is much smaller than those which were related to the other tissue-level predictors, implying that none of them is reliable for the analysis of contusion (Fig. 6).

The comparison of the DAI-related risk curves shows that the CSDM-based curves perform better (in terms of SSE) than the MPS-based curves (Table 4). This agrees with the previous observation that CSDM gives a better correlation with BrIC than MPS. The reliability analysis-based curves perform slightly better than the original curves which can be attributed to the above-noted advantages of the reliability analysis (Table 4). Other RIC- and RVCI-related curves have worse performance than other BrIC-based curves which could primarily come from the above-noted lower degree of correlation and the fact that these RIC- and RVCI-based curves are only estimations constructed for this comparison study based on available literature data and scaling techniques. This list of observations leads to the conclusion that the third hypothesis is also true.

As the BrIC gives a lower degree of correlation with RMDM and DDM than with the DAI-related predictors (Fig. 6), the obtained risk curves related to ASDH performs considerably worse $(\mathrm{SSE}=4.2379)$ than the best DAI-related curve (SSE $=0.3196$ ) (Table 5 ). The contusion-related curve gives negligible error in terms of SSE (SSE $=0.0074$ ) which primarily come from the negligible injury probabilities that were shown in Table 3. Unfortunately, the error of the ASDH-related risk curve appears in the curve related to the combined AIS 4+ injury (Eq. $(15 \mathrm{~b}))$ as well $(\mathrm{SSE}=4.0769)$. It is interesting, that the $\mathrm{HIC}_{15}$-related curve (Eq. (3)), which was obtained by reliability analysis [9], does not perform significantly worse in terms of SSE than the BrIC-related curve (Eq. (15b)), and performs much better than the original $\mathrm{HIC}_{15}$-related risk curve (Eq. (2)) which is currently applied in the standards. These ob- servations may also highlight the advantages of reliability analysis.

As a limitation of our study, it is noted that every tissue-level investigation which rely on FE simulation provides results which depend on the applied model to a certain extent. Several head models were applied in the literature [18], [5] which include different level of negligence (in terms of anatomic details, material behaviour, etc.). In most cases, these models are validated against the same experimental data and their results are in relatively good agreement [20], [4]. Nevertheless, all FE results could be somewhat different if other FE models were applied. It also means that the comparison of the injury metrics is biased in a sense that BrIC was originally constructed via the SIMon, while RIC and RVCI were proposed based on the results of other models.

Furthermore, in accordance with the recommendations of the authors of the improved SIMon model [18], the standard model version was applied the analyse ASDH and contusion. This model also went through rigorous validation procedure, but it is less detailed than the improved model, thus our results related to ASDH and contusion may be less reliable than the results related to DAI.

Although previous convergence study [9] showed that the selection and scaling of 100 tests seemed to be adequate for performing the reliability analysis, some deviation in the results of the comparison study may be expected if other records or more tests are considered.

Every FE reconstruction in this study belonged to a frontal test with $56 \mathrm{~km} / \mathrm{h}$ impact velocity (as per the regulations of the FMVSS 208 standard). Since different risk curves could belong to different crash scenarios (e.g., side impacts, other types of dummies, different impact velocities, etc.), theoretically, the currently obtained risk curves are valid to the above-noted test configurations only (the analysis of other crash scenarios may be an interesting task of the future studies).

The standardised Hybrid III dummy has excellent biofidelity and measurement capabilities, however, certain differences to humans still exist. As the load curves were derived from the acceleration time-histories measured on dummies, the observed results are based on the assumption that the above-mentioned difference does not cause a significant discrepancy in the calculated injury probabilities.

Although the correlation between the injury probabilities related to standardised tests and real-world crashes is affected by several factors, in the future, the currently obtained risk curves should be supported by the validation with real-world observations to help the 
decision-making regarding the new thresholds of the safety standards.

Some major sources of uncertainties have been considered in this study, however, other types of uncertainties (e.g., related to FE simulations, extremely large variability of the tissue properties [8], geometric and kinetic characteristics among humans, etc.) were not taken into account (the characterization of their effect may be an important task in future studies).

In the current analysis, the combined AIS 4+ curves were determined based on the considerations of three of the most common types of TBIs, however, other injury types (such as epidural hematoma) were not taken into account. Ideally, every possible injury type should be represented in the reliability analysis, and this may be the case in future studies when tissue-level thresholds become available for all injury types.

\section{Conclusions}

The tissue-level reliability analysis of the human brain for frontal car crashes resulted in slightly modified risk curves for DAI and new risk curves related to ASDH, contusion and combined AIS 4+ injury. Our results indicate that the probability of DAI should be primarily characterized by CSDM (instead of MPS) and the currently proposed risk curve (Eq. (13 b)). A moderate $R^{2}=0.61$ degree of correlation was observed for DAI, while considerably smaller values were found for the other injury types (Fig. 6). The results of the correlation analysis indicate that BrIC seems to be the best currently available injury metric, however, even better metrics may be found in the future, especially for the analysis of ASDH and contusion. Similarly, based on the evaluation of the risk curves (Tables 4, 5), the currently proposed BrIC-related risk curves seem to be the most reliable tool available for the evaluation of brain injury risk in case of frontal crash tests. However, these curves may be further improved in the future once new injury metrics and more robust tissue-level thresholds are developed for the different injury types.

\section{Acknowledgements}

This work was supported by the Higher Education Excellence Program of the Ministry of Human Capacities in the frame of Biotechnology research area of Budapest University of Technology and Economics (BME FIKP-BIO).

\section{References}

[1] Antona-Makoshi J., Traumatic Brain Injuries: Animal Experiments and Numerical Simulations to Support the Development of Brain Injury Criterion, Dissertation, Chalmers University of Technology, 2016.

[2] Dewan M.C., Rattani A., Gupta S., Baticulon R.E., Hung Y.-C., Punchak M., Agrawal A., Adeleye A.O., Shrime M.G., Rubiano A.M., Rosenfeld J.V., PARK K.B., Estimating the global incidence of traumatic brain injury, J. Neurosurg., 2019, 130, 1080-1097.

[3] EPANEChNiKov V.A., Nonparametric estimation of a multidimensional probability density, Theor. Probab. Appl., 1969, 14, 153-158.

[4] Gabler L.F., Crandall J.R., Panzer M.B., Assessment of Kinematic Brain Injury Metrics for Predicting Strain Responses in Diverse Automotive Impact Conditions, Ann. Biomed. Eng., 2016, 44 (12), 3705-3718.

[5] Gayzik F.S., Moreno D.P., Geer C.P., Wuertzer S.D., Martin R.S., Stitzel J.D., Development of a Full Body CAD Dataset for Computational Modeling: A Multimodality Approach, Ann. Biomed. Eng., 2011, 39 (10), 2568-2583.

[6] Gennarelli T.A., Wodzin E. (Eds.) Abbreviated injury scale 2005: update 2008, American Association for Automotive Medicine, 2008.

[7] Faul M., Xu L., Wald M.M., Coronado V.G., Traumatic Brain Injury in the United States: Emergency Department Visits, Hospitalizations and Deaths 2002-2006, National Center for Injury Prevention and Control, 2010, https://www.cdc.gov/ traumaticbraininjury/pdf/blue_book.pdf (accessed: 4 January 2021).

[8] Franceschini G., Bigoni D., Regitnig P., Holzapfel G.A., Brain tissue deforms similarly to filled elastomers and follows consolidation theory, Journal of the Mechanics and Physics of Solids, 2006, 54, 2592-2620.

[9] HAZAY M., DÉNES D., BOJTÁR I., The probability of traumatic brain injuries based on tissue-level reliability analysis, Acta Bioeng. Biomech., 2019, 21 (1), 141-152.

[10] Kameyama M., Karibe H., Onuma T., Tominaga T., Epidemiological study of head injury in Miyagi Neurotrauma Data Bank: Age, cause of injury, pathophysiology and outcome, Neurotraumatology, 2008, 31, 49-56 (in Japanese).

[11] Kimpara H., Iwamoto M., Mild Traumatic Brain Injury Predictors Based on Angular Accelerations During Impacts, Ann. Biomed. Eng., 2011, 40 (1), 114-126.

[12] Li X., Zhou Z., Kleiven S., An anatomically detailed and personalizable head injury model: Significance of brain and white matter tract morphological variability on strain, Biomech. Model Mechan., 2020, DOI: 10.1007/s10237020-01391-8.

[13] National Highway Traffic Safety Administration, Final economic assessment, FMVSS No. 201, upper interior head protection, U.S. Department of Transportation, 1995, https:// www.regulations.gov/document?D=NHTSA-1996-1762-0003 (accessed: 4 January 2021).

[14] National Highway Traffic Safety Administration, Federal Motor Vehicle Safety Standards 208, Occupant Crash Protection, U.S. Department of Transportation, 2019, https:// ecfr.federalregister.gov/current/title-49/subtitle-B/chapter-V/ part-571/subpart-B/section-571.208 (accessed: 4 January 2021). 
[15] National Highway Traffic Safety Administration, New Car Assessment Program, U.S. Department of Transportation, 2015, https://www.govinfo.gov/content/pkg/FR-2015-12-16/pdf/ 2015-31323.pdf (accessed: 4 January 2021).

[16] Prasad P., Mertz H.J., The position of the United States delegation to the ISO working group 6 on the use of HIC in the automotive environment, Society of Automotive Engineers, Technical Paper No. 851246, 1985, DOI: 10.4271/851246.

[17] TakHounTs E.G., EPPINGER R.H., CAMPBell J.Q., TANNOUS R.E., POWER E.D., SHOOK L.S., On the development of the SIMon finite element head model, 47th Stapp. Car Crash J., 2003, 47, 107-133.

[18] Takhounts E.G., Ridella S.A., Hasija V., Tannous R.E., Campbell J.Q., Malone D., Danelson K., Stitzel J., Rowson S., DuMA S., Investigation of Traumatic Brain Injuries Using the Next Generation of Simulated Injury Monitor (SIMon) Finite Element Head Model, Stapp. Car Crash J., 2008, 52, 1-31.

[19] Takhounts E.G., Hasija V., Eppinger R.H., Analysis of $3 D$ rigid body motion using the nine acceleration array and the randomly distributed in-plane accelerometer systems, Proceedings of the $21 \mathrm{st}$ (ESV) International Technical Conference on the Enhanced Safety of Vehicles, 2009, https://www-esv.nhtsa.dot.gov/Proceedings/21/09-0402.pdf (accessed: 5 January 2021).
[20] Takhounts E.G., Craig M.J., Moorhouse K., McFadden J., Hasija V., Development of brain injury criteria (BrIC), Stapp. Car Crash J., 2013, 57, 243-266.

[21] The MathWorks, MATLAB Programming Fundamentals 2020b, The MathWorks, 2020, https://www.mathworks.com/ help/pdf_doc/matlab/matlab_prog.pdf (accessed: 5 January 2021).

[22] United Nations Economics Commission for Europe, Regulation No 94 of the Economics Commission for Europe of the United Nations (UNECE) - Uniform provisions concerning the approval of vehicles with regard to the protection of the occupants in the event of a frontal collision [2018/178], Official Journal of the European Union, Legislation 35, 2018, 61, 1-60.

[23] Versace J., A review of the severity index, Proceedings of the 15th Stapp Car Crash Conference, 1971, DOI: 10.4271/710881.

[24] Wilhelm J., Ptak M., Fernandes F.A.O., Kubicki K., Kwiatkowski A., Ratajczak M., Sawicki M., Szarek D., Injury Biomechanics of a Child's Head: Problems, Challenges and Possibilities with a New aHEAD Finite Element Model, Appl. Sci., 2020, 10, 4467.

[25] YANAOKA T., DOKKO Y., TAKAHASHI Y., Investigation on an Injury Criterion Related to Traumatic Brain Injury Primarily Induced by Head Rotation, Society of Automotive Engineers, Technical Paper No. 2015-01-1439, 2015, DOI: 10.4271/201501-1439. 